\title{
Flight Mechanics Feasibility Assessment for Co-Delivery of Direct-Entry Probe and Aerocapture Orbiter
}

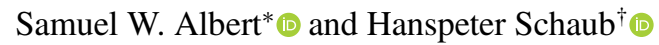 \\ University of Colorado Boulder, Boulder, Colorado 80303 \\ and \\ Robert D. Braun辛 \\ California Institute of Technology, Pasadena, California 91125 \\ https://doi.org/10.2514/1.A34953
}

\begin{abstract}
The co-delivery of a direct-entry probe and an aerocapture orbiter from a single atmospheric entry state is a novel way to include ride-along probes or orbiters on interplanetary missions. This is made possible through combining two technologies: low-cost small satellites and aerocapture. This study investigates the feasibility of this co-delivery method from a flight-mechanics perspective. The availability of direct-entry and aerocapture trajectories from a single entry flight-path angle is assessed for a large range of feasible ballistic coefficients at Earth, Mars, Venus, Titan, and Neptune. Apoapsis altitude, peak heat flux, total heat load, and peak g-load are also quantified across this trade space. A representative scenario implementing closed-loop guidance is presented for a proof of concept, and the trajectory dispersions due to relevant uncertainties are quantified in a Monte Carlo analysis. Passive ballistic impactor or penetrator probes as a secondary mission with a primary lift-modulated aerocapture orbiter is identified as the most promising configuration.
\end{abstract}

\section{Introduction}

$\mathbf{C}$ O-DELIVERY of a probe and an orbiter is a powerful architecture for a variety of interplanetary missions. The Galileo and Cassini-Huygens missions are two famous examples, among many others, of this approach. Given the infrequency of major planetary science missions, it is desirable to maximize scientific return by gathering data from orbit as well as in situ measurements from the atmosphere or surface. Although interplanetary probe and orbiter missions have already been accomplished a number of times, two technologies could be combined to enable a new type of co-delivery architecture for planetary science missions.

The first technology is low-cost small satellites (SmallSats), especially CubeSats, which have accounted for an increasingly large share of satellites launched each year since around 2012 [1]. Technological innovations, including the miniaturization of electronics and availability of commercial off-the-shelf hardware, have led to a steady increase in the capabilities possible in these small form factors, and CubeSat missions have now moved beyond serving a primarily educational role to make numerous notable scientific contributions [2]. A 2014 study sponsored by the Keck Institute for Space Studies presented space science mission concepts "uniquely enabled by the small satellite platform," and recommended including small spacecraft as secondaries on all missions beyond low Earth orbit [3]. NASA has also studied a variety of mission concepts through its Planetary Science Deep Space SmallSat Studies program [4]. In November 2018, Mars Cube One (MarCO)-A and MarCO-B, the twin CubeSat communications relays accompanying the InSight Mars lander, successfully demonstrated the merit of SmallSats in deep space applications [5] . Ongoing

Received 8 September 2020; revision received 9 June 2021; accepted for publication 28 June 2021; published online 9 September 2021. Copyright (C) 2021 by Samuel W. Albert. Published by the American Institute of Aeronautics and Astronautics, Inc., with permission. All requests for copying and permission to reprint should be submitted to CCC at www.copyright.com; employ the eISSN 1533-6794 to initiate your request. See also AIAA Rights and Permissions www.aiaa.org/randp.

*Ph.D. Student, Ann and H.J. Smead Aerospace Engineering Sciences, 3775 Discovery Drive, 429 UCB-CCAR. Student Member AIAA.

${ }^{\dagger}$ Glenn L. Murphy Chair of Engineering, Ann and H.J. Smead Aerospace Engineering Sciences, 3775 Discovery Drive, 429 UCB-CCAR. Fellow AIAA.

${ }^{\sharp}$ Bren Professor of Aerospace, Graduate Aerospace Laboratories, 1200 E. California Boulevard, MC MC 105-50. Fellow AIAA. research is applying SmallSat innovation to entry, descent, and landing (EDL) by developing technologies including deployable aeroshells and multifunctional EDL structures [6,7]. SmallSat secondary spacecraft enhance planetary science missions only if the secondary mission can minimize the additional mass, risk, cost, and complexity to the primary mission.

The second technology is aerocapture, the often-studied technique of flying through the atmosphere of a planet to reduce the spacecraft's energy and capture into orbit, as shown in Fig. 1. This technique has been studied for decades, but not implemented in flight. In recent years, significant work has contributed to the development of aerocapture and related technologies, including development of advanced thermal protection systems [8], robust flight-control methods and guidance algorithms [9-11], uncertainty quantification [12-15], deployable decelerator technology $[\underline{6}, \underline{16}, \underline{17}]$, and broad aerocapture technology studies [18-20] to list a few. A 2016 study at the NASA Jet Propulsion Laboratory concluded that, while aerocapture technology readiness is destination dependent, no prior flight demonstration would be needed to implement aerocapture on Titan, Mars, and possibly Venus [18]. Some of the renewed interest in aerocapture can be attributed to recent concepts for missions to the ice giants (Uranus and Neptune) in preparation for the Planetary Science Decadal Survey [21], because it is these destinations where aerocapture can offer the most benefit compared to propulsive orbit insertion [22].

The concept that combines secondary SmallSats and aerocapture is to design a probe and an orbiter to reach their desired final states from a single approach trajectory and entry state, illustrated in Fig. 2. The two vehicles travel together during cruise and separate shortly before atmospheric entry, and then diverge during atmospheric flight due to differences in their aerodynamic properties and control strategies. The orbiter stays higher in the atmosphere, dissipating just enough energy to perform aerocapture, while the probe continues deeper into the atmosphere until reaching its desired target state, such as parachute deployment or surface impact. By designing the probe and orbiter to target a single atmospheric entry state, the need for a critical divert maneuver performed shortly before entry is avoided. For example, a satellite using lift-modulated aerocapture to reach Mars orbit could release several small probes that follow ballistic trajectories down to the surface. A jettison event is still required to physically separate the orbiter and probe and prevent recontact in the atmosphere, akin to the mechanical separation of the Mars Science Laboratory (MSL) aeroshell from its cruise stage $10 \mathrm{~min}$ before atmospheric entry [23]. In general, this co-delivery approach can apply to missions with multiple 


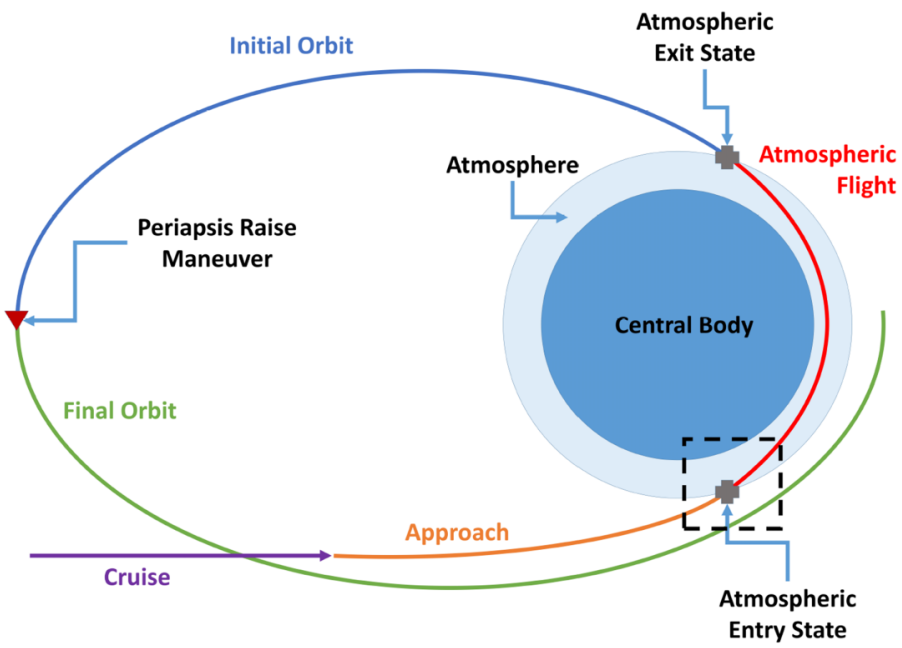

Fig. 1 Diagram of the aerocapture process.

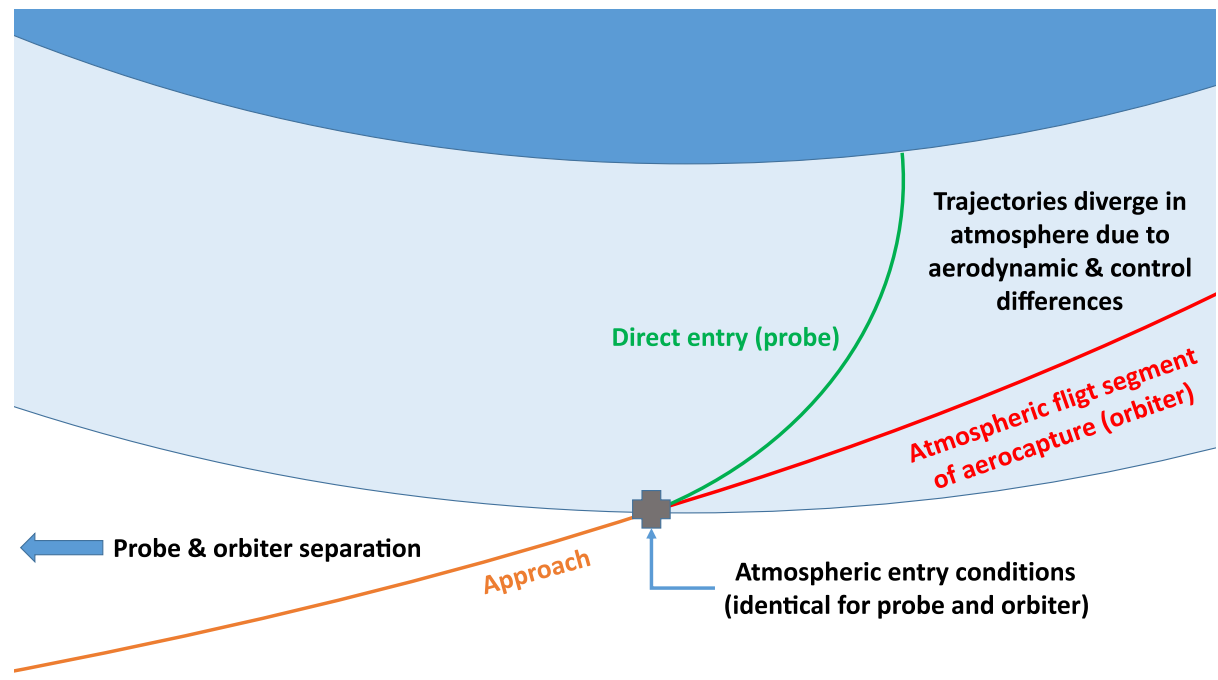

Fig. 2 Conceptual diagram of co-delivery from a single entry state, shown as a close-up view of the region in the dashed-line box in Fig. 1 ; features exaggerated.

probes or orbiters, but for simplicity this study proceeds assuming only one of each. The following are key terms used in this study:

1) "Co-delivery" refers to any two or more spacecraft that reach a shared destination via a single interplanetary trajectory, such as the delivery of five separate probes by the Pioneer Venus Multiprobe bus [24].

2) "Probe" is used as a catch-all term, including landers, impactors, deep atmospheric probes, etc.

3) "Secondary" refers to a smaller, ride-along addition to a larger, more expensive "primary" craft; for example, MarCO was a secondary mission for the InSight primary spacecraft. In the context of the proposed co-delivery method, a primary orbiter could have a secondary probe or the other way around; hence, these terms are defined separately.

The primary motivation for targeting a single entry state for both orbiter and probe is to avoid requiring a divert maneuver, and managing its associated error, shortly before entry. If this maneuver is performed early, the probe would either require a propulsion subsystem and navigation capability, or would be coasting without course-correction capability from separation until entry. The later the orbiter performs the divert maneuver, the larger this maneuver becomes and the less time there is to quantify and potentially mitigate maneuver execution error. This is not to say that these other codelivery architectures are not feasible; indeed, Galileo and CassiniHuygens successfully had probes coast passively for nearly 150 and 20 days, respectively $[25,26]$. Rather, targeting a single entry condition is a solution to this tradeoff that reduces maneuver complexity on approach and eliminates a source of navigation error. Furthermore, by codelivering the probe and orbiter, the secondary craft is able to benefit from the resources of the primary spacecraft, such as power, propulsion, and communications, until shortly before atmospheric entry. This may significantly simplify the design of the secondary ride-along craft compared to separate post-launch operations; for example, independent operations and navigation during cruise proved to be a significant challenge for the MarCO CubeSats [27].

Reference [28] qualitatively discusses the challenges of the proposed co-delivery method in detail. These challenges include the timing and dynamics of the separation event, post-separation collision concerns, timing and observation geometry between the orbiter and probe, and the feasibility of trajectories that deliver an orbiter for aerocapture and a probe for direct entry from a single entry state. The last of these is the focus of this study, with the remaining challenges left for future work.

This study focuses on the feasibility of the flight mechanics associated with this co-delivery strategy. A broad trade space is explored to understand the regions of feasibility for co-delivery from a single entry state while quantifying relevant mission constraints. Earth, Mars, Venus, Titan, and Neptune applications are considered. A single representative scenario is developed that implements closed-loop guidance for both vehicles, and illustrates the performance of these vehicles under relevant uncertainties via Monte Carlo simulation. 


\section{Trade Study}

The purpose of this section is to understand, at a high level, the combinations of trajectories and vehicles for which co-delivery from a single entry state is a possibility. A wide range of entry trajectories are simulated and classified by their final states, and a number of key constraining parameters are computed. The goal of this study is to demonstrate the fundamental flight mechanics feasibility of this co-delivery method at each destination and provide a starting point for further investigation of any specific mission concept.

\section{A. Methodology}

To simulate these trajectories, three-degree-of-freedom equations of motion are numerically integrated assuming a point-mass gravity with lift and drag forces acting on each vehicle [29]. Consistent with the flight of a blunt body in hypersonic continuum flow, constant aerodynamic coefficients, constant mass, and zero thrust are assumed, as well as zero wind. The vehicle state is propagated using a variable-step Runge-Kutta numerical integration method of order 5(4) [30]. The vehicle is initialized at the atmospheric interface altitude $h_{\text {atm }}$, and the values used are listed in Table 1 [34-36,38]. For each target destination, a representative planet-relative entry velocity $V_{R, 0}$ is defined based on entry velocities of previous planetary entry missions or aerocapture mission studies [31-33]. Entry flight-path angle (EFPA) and ballistic coefficient are varied as part of the trade study. Entry flight-path angle is the angle between the planet-relative velocity vector of the vehicle at atmospheric entry and the local horizontal. The ballistic coefficient $\beta$ is effectively a ratio of inertial to aerodynamic forces on the vehicle and is defined in Eq. (1), where $m$ is vehicle mass, $C_{D}$ is hypersonic drag coefficient, and $A$ is reference area. Note that, although the particular results described herein will vary as a function of entry velocity, the purpose of this work is to demonstrate the conceptual feasibility of this co-delivery technique. The parameters used in this analysis are listed in Table $\underline{1}$.

$$
\beta=\frac{m}{C_{D} A}
$$

Profiles of atmospheric density are taken from the nominal output of the Global Reference Atmospheric Model (GRAM) for that planet/ moon [40-44], where each GRAM provides an engineering-level model that can produce both mean and dispersed atmospheric data. The density profile is then linearly interpolated with altitude; although density varies approximately exponentially with altitude, GRAM data are output every $0.1 \mathrm{~km}$, and so linear interpolation between data points is sufficiently accurate for this application. To approximately characterize the effect of density variability, results are shown for density profiles at \pm 3 standard deviations from nominal, where these $\pm 3 \sigma$ profiles are directly output by GRAM.

Several potentially constraining quantities are calculated for each trajectory, one of which is peak heat flux. Specifically, the peak convective heat flux at the stagnation point for a fully catalytic surface is estimated using the Sutton-Graves method [45] in the form shown

\section{Table 1 Relevant planetary constants}

\begin{tabular}{lcccc}
\hline \hline $\begin{array}{l}\text { Central } \\
\text { body }\end{array}$ & $h_{\text {atm }, \mathrm{km}}$ & $\begin{array}{c}V_{R, 0}, \\
\mathrm{~km} / \mathrm{s}\end{array}$ & $k, \mathrm{~kg}^{0.5} / \mathrm{m}$ & $\begin{array}{c}\text { Atmospheric composition } \\
\text { by volume }\end{array}$ \\
\hline Earth & $125[\underline{34}]^{\mathrm{a}}$ & 11 & $1.748 \times 10^{-4}$ & $\begin{array}{c}78.1 \% \mathrm{~N}_{2} ; 20.9 \% \mathrm{O}_{2}[\underline{35}] \\
\text { Mars }\end{array}$ \\
$125[\underline{36}]$ & 6 & $1.904 \times 10^{-4}$ & $\begin{array}{c}2.59 \% \mathrm{~N}_{2} ; 95.1 \% \mathrm{CO}_{2} ; \\
1.94 \% \mathrm{Ar}[\underline{37}]\end{array}$ \\
Venus & $135[\underline{35}]$ & 11.5 & $1.897 \times 10^{-04}$ & $\begin{array}{c}3.50 \% \mathrm{~N}_{2} ; 96.5 \% \\
\mathrm{CO}_{2}[\underline{35}]\end{array}$ \\
Titan & $800[\underline{35}]$ & 6 & $1.758 \times 10^{-04}$ & $\begin{array}{c}97.7 \% \mathrm{~N}_{2} ; 2.30 \% \\
\mathrm{CH}_{4}[\underline{35}]\end{array}$ \\
Neptune & $1000[\underline{38}]$ & 27 & $7.361 \times 10^{-05}$ & $\begin{array}{c}1.50 \% \mathrm{CH}_{4} ; 79.6 \% \mathrm{H}_{2} ; \\
18.9 \% \mathrm{He}[\underline{39}]^{\mathrm{b}}\end{array}$ \\
\hline \hline
\end{tabular}

${ }^{\mathrm{a} O r i o n}$ uses $h_{\mathrm{atm}}=400,000 \mathrm{ft}$, which here is rounded up to $125 \mathrm{~km}$ to match convention. bParticular values chosen to match NASA Space Science Data Coordinated Archive fact sheet for Neptune. in Eq. (2). In this equation, $\rho$ is density and $R_{n}$ is effective nose radius, and the $k$ coefficients and the atmospheric composition data used to compute them are listed in Table $1[35,37,39]$. Radiative heating is not included in this analysis. Total integrated heat load is computed by numerically integrating the stagnation point convective heat flux over time. An effective nose radius of $R_{n}=1 \mathrm{~m}$ is assumed, which allows easy scaling of these heating results for other nose radius values. The maximum sensed acceleration in terms of Earth g's values is also calculated.

$$
\dot{q}_{s}=k \sqrt{\frac{\rho}{R_{n}}} V_{R}^{3}
$$

Each trajectory is categorized based on its exit state: if the trajectory intersects the surface (or some minimum altitude), it is a probe; if the vehicle exits the atmosphere on an elliptical orbit, it is an orbiter; and if the vehicle exits the atmosphere on a hyperbolic orbit, the trajectory is categorized as escape. For the orbiter trajectories, apoapsis altitude is computed using the post-atmospheric Keplerian state of the vehicle.

Three types of trajectories are described in the open-loop analysis presented in this study: ballistic, full-lift-up, and full-lift-down. These descriptors do not imply that the vehicle has no additional control authority; rather, they represent nominal trajectories for which no liftor drag-modulation is required. A lift-to-drag ratio of $L / D=0.25$ is selected based on the approximate hypersonic trim $L / D$ of MSL and Mars 2020 and the known capabilities of a 70 deg sphere cone aeroshell [36]. Although the results of this study provide insight into the consequences of increasing or decreasing $L / D$ from this value, quantitative analysis for vehicles with significantly different $L / D$ is left for future work. By showing these three cases, the set of trajectories approximately accessible with a $70 \mathrm{deg}$ sphere cone aeroshell is characterized for each scenario.

\section{B. Results}

The results at each planetary destination are summarized in Figs. 3-7. For each of the three trajectory types, trajectories are simulated across a grid of varying EFPA and ballistic coefficient. For each grid, at any given $\beta$, there will be some EFPA value that delineates between probes and orbiters. These EFPA values form the black line on each plot. Similarly, if the inertial entry velocity exceeds escape velocity, there will be an EFPA value delineating between orbiters and escape trajectories, and this is shown as the purple line. The shaded regions for each line are bounded by the values of that line when the $\pm 3 \sigma$ profiles are used for density. Therefore, any grid points left of the black line are probe trajectories, any grid points between the lines are orbiters (aerocapture), and to the right of the purple line are escape trajectories. Contours of apoapsis altitude, peak g-load, peak heat flux, and total heat load are then overlaid for each plot. Note that the contour values are not necessarily evenly incremented, and that the $x$-axis scale varies significantly between destinations.

The interpretation of these plots is illustrated through the following example. By definition, the proposed co-delivery method is feasible where a probe trajectory and orbiter trajectory both exist at the same EFPA for realistic ballistic coefficients. Because the vehicles share an entry condition, co-delivery scenarios are identified in these plots with vertical cross sections along a single EFPA. As a simple example, a vertical line at $-5.5 \mathrm{deg}$ (not shown) for the Earth-ballistic plot would pass through the middle of the black line. Here, ballistic coefficients less than $75 \mathrm{~kg} \cdot \mathrm{m}^{-2}$ are probes and greater than $110 \mathrm{~kg} \cdot \mathrm{m}^{-2}$ are orbiters. Thus, for $11 \mathrm{~km} / \mathrm{s}$ entry at Earth with an EFPA of $-5.5 \mathrm{deg}$, co-delivery from a single entry state is possible using only ballistic trajectories, just by tuning the $\beta$ values of the two vehicles.

The application of lift broadens this feasible range significantly. In Fig. 3, a light blue vertical line is added at a nominal EFPA of $-6.2 \overline{5} \mathrm{deg}$. On the ballistic plot, the line is entirely behind the orbiters/probes cutoff, meaning all ballistic coefficients in the range considered $\left(10-200 \mathrm{~kg} \cdot \mathrm{m}^{-2}\right)$ result in probe trajectories. On the full-liftup plot, the line is entirely in front of the cutoff line, and so all $\beta$ values 


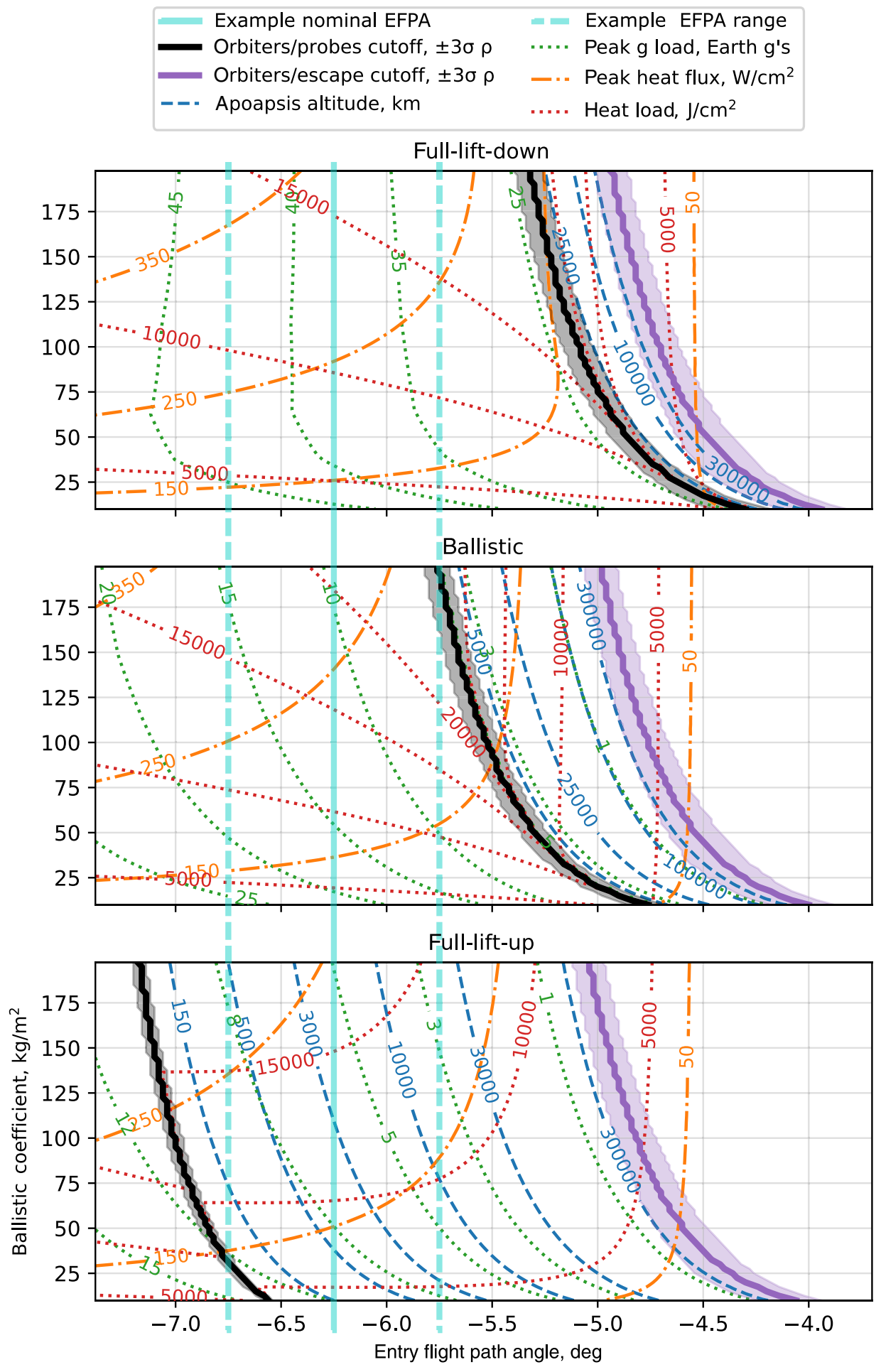

Fig. 3 Feasibility space for Earth, $11 \mathrm{~km} \cdot \mathrm{s}^{-1}$ relative entry velocity, shown with example annotation.

result in orbiter trajectories. The initial apoapsis altitudes for these trajectories vary with ballistic coefficient and are shown in the dashed blue contour lines.

For the proposed co-delivery method to be plausible, the architecture should be robust to a number of uncertainties, including navigation uncertainty. This can be described as an entry corridor, a range of possible EFPA values. In Fig. 3 , the dashed light blue vertical lines represent an entry corridor of $-\overline{6} .25 \pm 0.5 \mathrm{deg}$. As a result of this uncertainty, the dashed lines now intersect the black cutoff lines for ballistic and lift-up trajectories, and these intersection points give the ballistic coefficient requirements for this scenario. For feasibility even with this large EFPA uncertainty, the orbiter $\beta$ would need to be at least $40 \mathrm{~kg} \cdot \mathrm{m}^{-2}$ and the probe coefficient no greater than $160 \mathrm{~kg} \cdot \mathrm{m}^{-2}$. The value of $\pm 0.5 \mathrm{deg}$ used here is only an example; the same process can be followed for any width entry corridor using the results in Figs. 3-7.

These ballistic coefficients might be further constrained by other requirements. Continuing the example annotated in Fig. 3 , to achieve an initial apoapsis altitude of at least $150 \mathrm{~km}$, the orbiter $\beta$ should be at least $75 \mathrm{~kg} \cdot \mathrm{m}^{-2}$. To keep the total heat load at the stagnation point below $20 \mathrm{~kJ} \cdot \mathrm{cm}^{-2}$, the probe $\beta$ should be no greater than $120 \mathrm{~kg} \cdot \mathrm{m}^{-2}$. Additionally, note that the EFPA range still does not intersect the cutoff line on the full-lift-down plot, and so any ballistic coefficient in range would result in a probe trajectory, although the peak g-loads are 

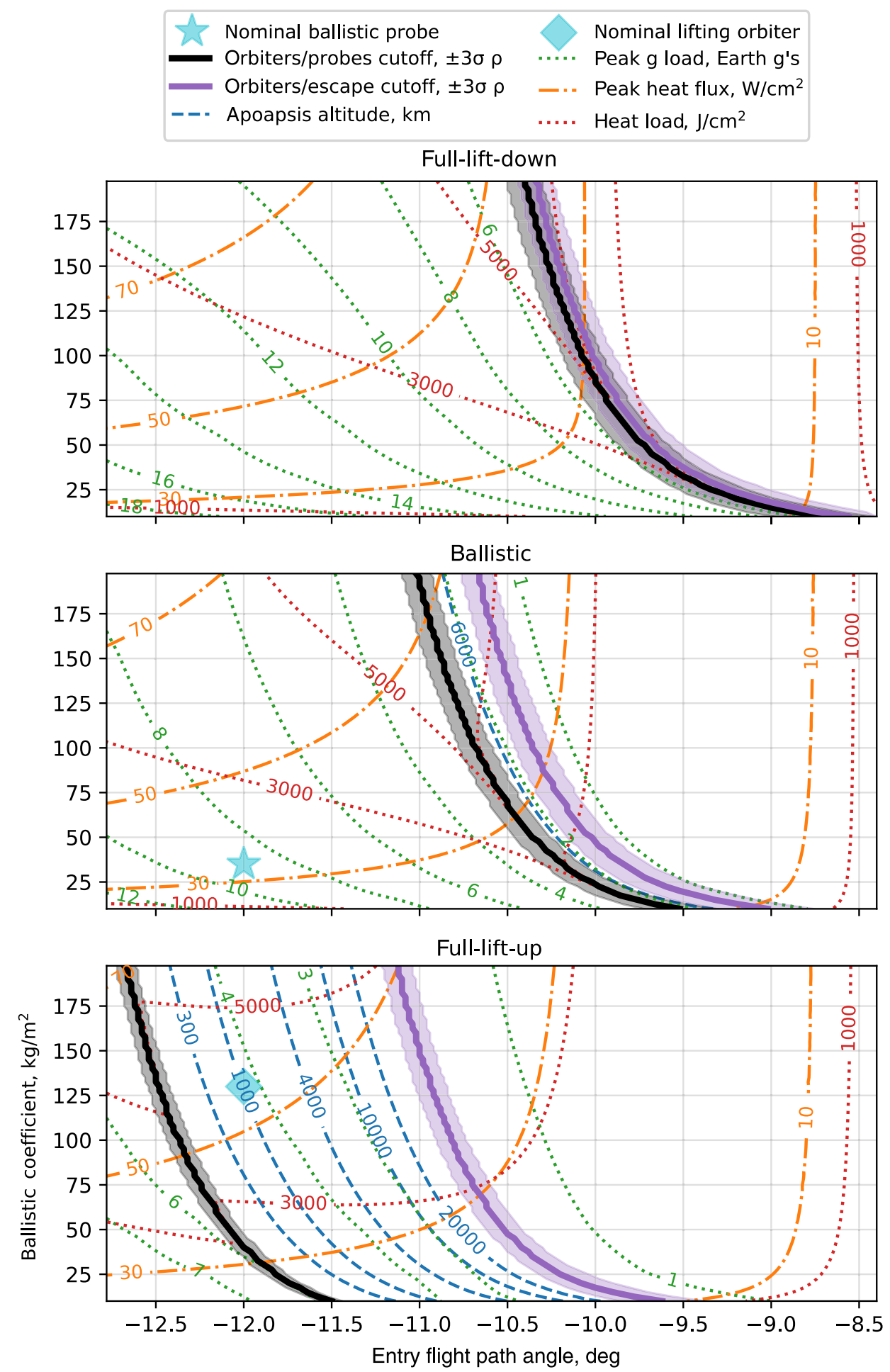

Fig. 4 Feasibility space for Mars, $6 \mathrm{~km} \cdot \mathrm{s}^{-1}$ relative entry velocity, and nominal scenario used in Sec. III marked in blue.

significantly higher for lift-down trajectories. In addition to these flight mechanics constraints, packaging and vehicle geometry considerations make some ballistic coefficients more feasible than others.

The preceding example demonstrates how a mission designer can choose constraints on nominal EFPA, entry corridor, apoapsis, etc., and then directly assess the feasibility of probe and orbiter co-delivery from a single entry state for that mission scenario from the plots in Figs. $\underline{3}-\mathbf{7}$.

\section{Discussion}

The feasibility assessment at each destination depends on the specific scenario and constraints, making it challenging to compare the destinations in a general way. One heuristic approach is to consider the EFPA range spanned by the probes/orbiters cutoff line (i.e., the difference between the probe/orbiter transition EFPAs at $\beta=$ $200 \mathrm{~kg} \cdot \mathrm{m}^{-2}$ and $\beta=10 \mathrm{~kg} \cdot \mathrm{m}^{-2}$ for ballistic trajectories, including the narrowing effect of the atmospheric uncertainty bars). In ascending order, this value is approximately $0.66 \mathrm{deg}$ at Venus, $0.84 \mathrm{deg}$ at Earth, $0.88 \mathrm{deg}$ at Neptune, $1.22 \mathrm{deg}$ at Mars, and $3.8 \mathrm{deg}$ at Titan. These values reflect the ranges of usable EFPAs for two ballistic vehicles, meaning Titan is by far the most flexible if no nominal lift is required. A similar heuristic parameter is the EFPA range gained from a full-lift-up trajectory, defined as the difference between the probe/orbiter transition EFPAs at $\beta=100 \mathrm{~kg} \cdot \mathrm{m}^{-2}$ for full-lift-up and ballistic trajectories, including the narrowing effect of 


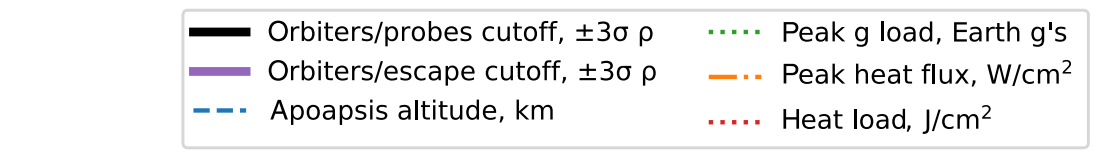
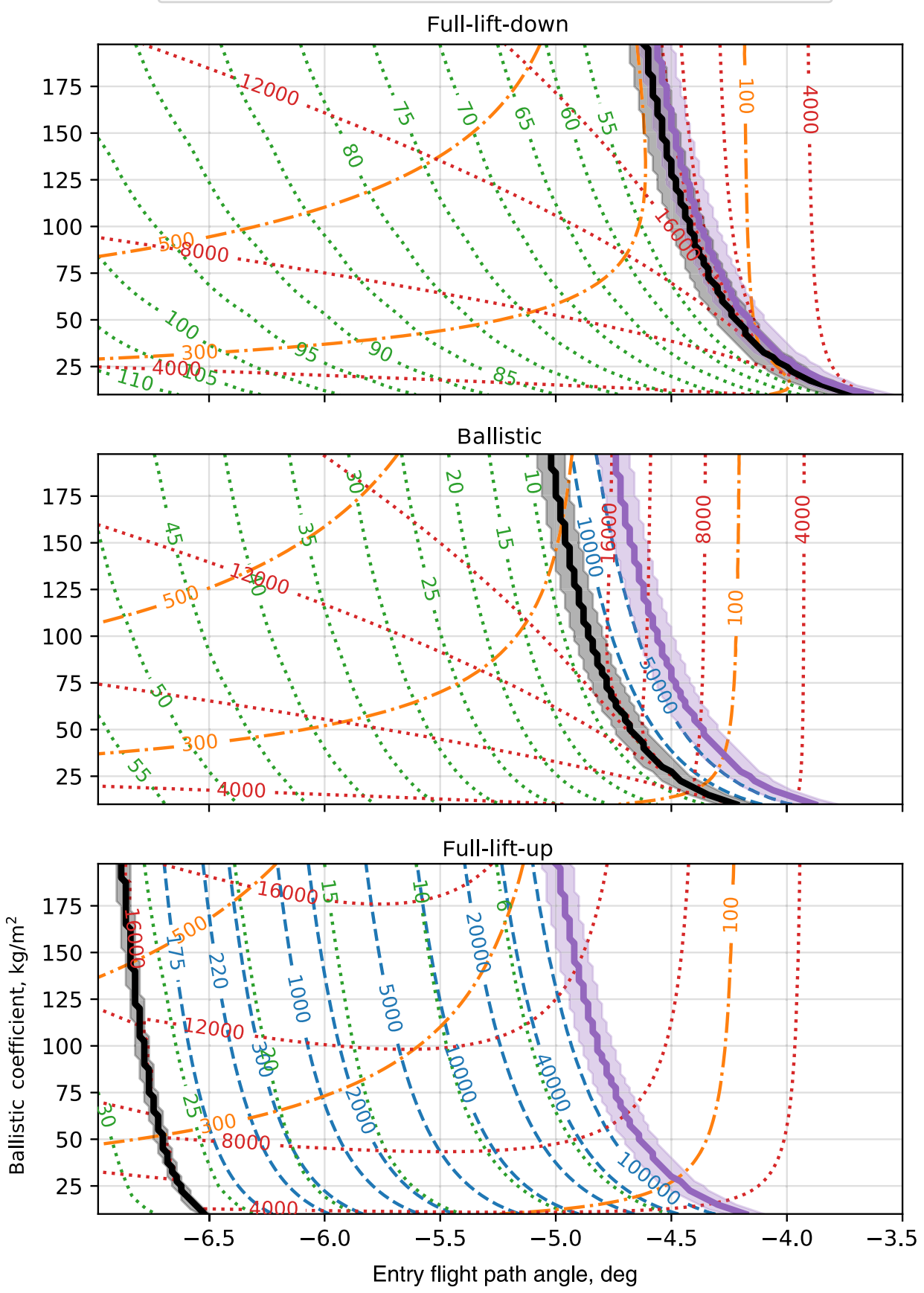

Fig. 5 Feasibility space for Venus, $11.5 \mathrm{~km} \cdot \mathrm{s}^{-1}$ relative entry velocity.

the atmospheric uncertainty bars. Again, in ascending order, this value is approximately $1.18 \mathrm{deg}$ at Neptune, $1.36 \mathrm{deg}$ at Earth, $1.52 \mathrm{deg}$ at Mars, $1.84 \mathrm{deg}$ at Venus, and $1.9 \mathrm{deg}$ at Titan. Titan again has the widest range by this measure, although by a smaller margin. Notably, Venus has the smallest range for ballistic-only trajectories but has the second-widest range by this measure of lift effectiveness. The small scale height of the Venusian atmosphere at aerocapture altitudes corresponds to rapid density variations with altitude [32], resulting in narrow corridor widths but a large control authority for lifting vehicles. Furthermore, all else being equal, high entry velocities lead to larger theoretical corridor widths for lift-modulation aerocapture vehicles [46], and the representative entry velocity chosen for Venus in this study is high relative to the mass of the planet. It is important to note that these benefits are directly traded off by high g-loads, heat fluxes, and heat loads at Venus; the high entry velocity at Neptune, dictated by its large gravity well and the constraint of reasonable times of flight from Earth, has similar drawbacks. Note that the particular values of these EFPA ranges are tied to the choices of atmospheric interface altitudes listed in Table 1.

The results shown in Figs. 3-7 and discussed previously are primarily in terms of ballistic coefficient, which is a ratio and provides no information on the actual mass and volume of the vehicle. The mechanical and aerodynamic design of specific aeroshells to meet a target ballistic coefficient, fit within secondary SmallSat mass and volume constraints, and accommodate a science payload is beyond the scope of this study. That said, there is precedent for entry 


\begin{tabular}{|c|c|}
\hline & \\
\hline $\begin{array}{l}\text { Orbiters/probes cutoff, } \pm 3 \sigma \rho \\
\text { Orbiters/escape cutoff, } \pm 3 \sigma \rho \\
\text {--. Apoapsis altitude, } \mathrm{km}\end{array}$ & $\begin{array}{l}\ldots . . \text { Peak g load, Earth g's } \\
\ldots .\end{array}$ \\
\hline
\end{tabular}
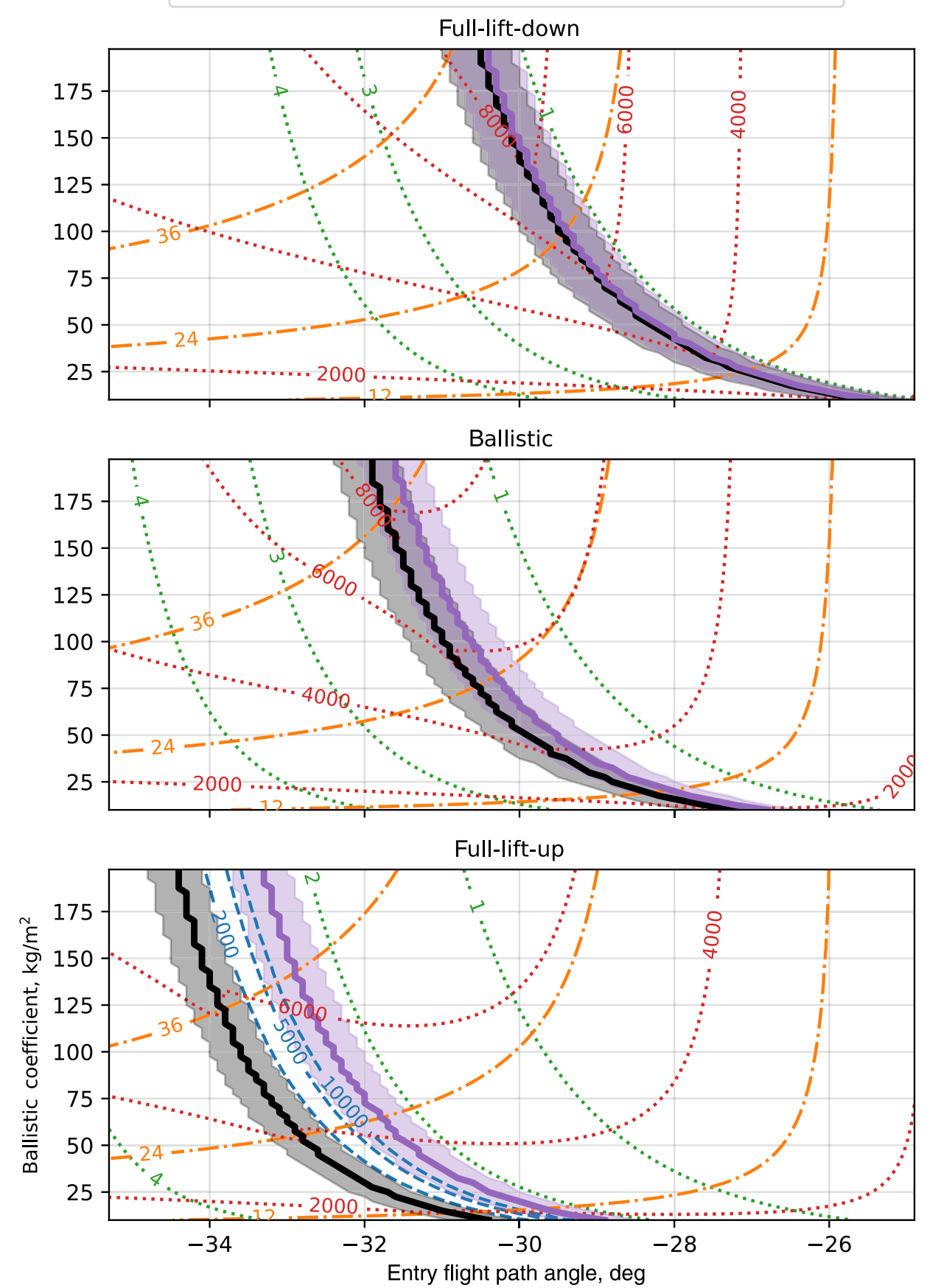

Fig. 6 Feasibility space for Titan, $6 \mathrm{~km} \cdot \mathrm{s}^{-1}$ relative entry velocity.

capsules in a SmallSat form factor. Most notably, the Mars Microprobes provide flight heritage at Mars, and each probe had a ballistic coefficient of $35.6 \mathrm{~kg} \cdot \mathrm{m}^{-2}$ and an entry mass of $3.6 \mathrm{~kg}$, and would have fit within a $35 \times 35 \times 27.5 \mathrm{~cm}$ box [47], well within the mass and volume constraints for a secondary payload on an Evolved Expendable Launch Vehicle Secondary Payload Adapter (ESPA) ring [48]. The Adaptive Deployable Entry and Placement Technology deployable aeroshell enables aeroshell diameters of up to $1.7 \mathrm{~m}$ to stow within an ESPA secondary payload volume, and has an expected ballistic coefficient in the range of $25-50 \mathrm{~kg} \cdot \mathrm{m}^{-2}$ and entry mass of $75-150 \mathrm{~kg}$ for delivery of a $12 \mathrm{U}$ CubeSat payload volume [6]. The notional design for the Small High Impact Energy Landing Device has a ballistic coefficient as low as $10 \mathrm{~kg} \cdot \mathrm{m}^{-2}$ for an entry mass of $50 \mathrm{~kg}$ and $6 \mathrm{~kg}$ payload mass $[7,49]$. These example designs are included here to illustrate the fact that, although detailed design is out of scope, the co-delivery method is conceivable with SmallSat-class vehicles.

There are some key limitations to the approach taken in this study. For the sake of space, only one entry velocity is considered for each destination. In general, increases in entry velocity on the order of $1 \mathrm{~km} \cdot \mathrm{s}^{-1}$ lead to an increase in lift-modulation control authority, increased g-loads, and a compression of the available apoapsis radii in terms of EFPA, but the overall feasibility of co-delivery is not dramatically affected. This is shown in Ref. [50] through a comparison of feasibility with 10,11 , and $12.5 \mathrm{~km} \cdot \mathrm{s}^{-1}$ entry velocities at Earth. Another limitation is the bounding case approach to lift modulation. 

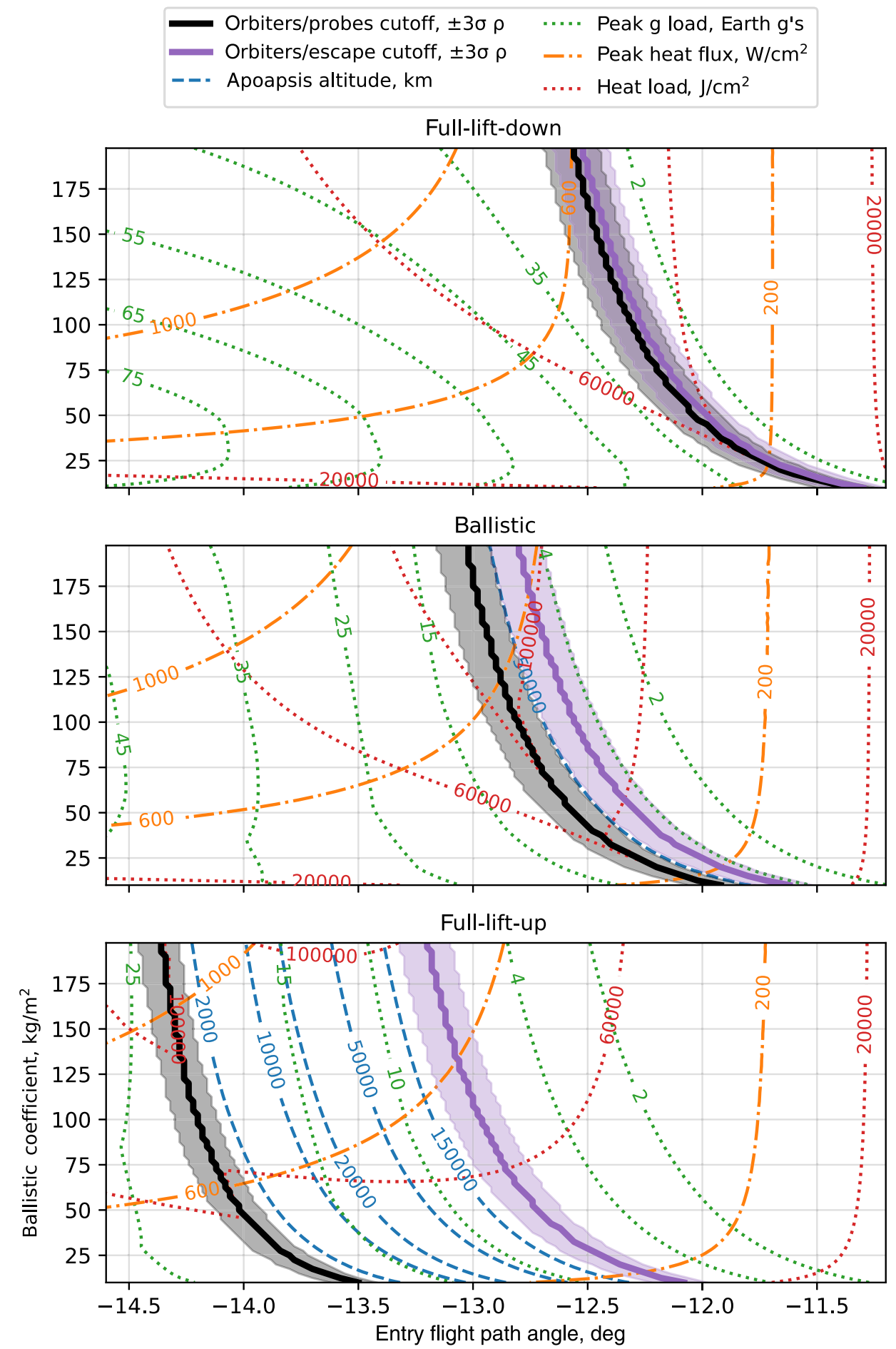

Fig. 7 Feasibility space for Neptune, $27 \mathrm{~km} \cdot \mathrm{s}^{-1}$ relative entry velocity.

Although it is possible to use $100 \%$ of available lift to bias the nominal trajectory (e.g., Viking flew a full-lift-up trajectory with no guidance), in general some control authority must be allocated to compensate for uncertainties in EFPA, atmospheric density, vehicle parameters, etc. MSL, for example, used about $70 \%$ of its available lift to bias its nominal trajectory, reserving $30 \%$ for control authority margin [36]. The ability of the results shown here to capture these types of trajectories is limited. For example, for a lift-up trajectory at Earth, $11 \mathrm{~km} \cdot \mathrm{s}^{-1}$, and an EFPA of $-6 \mathrm{deg}$, a ballistic coefficient of $50 \mathrm{~kg}$. $\mathrm{m}^{-2}$ results in an apoapsis altitude of about $3000 \mathrm{~km}$. Intuitively, a similar trajectory that instead uses only $70 \%$ of its lift for the nominal trajectory would result in aerocapture with a lower apoapsis, but the results shown here do not quantify this idea. Nonetheless, these results give bounding cases within which a vehicle could reserve some control margin for uncertainties by targeting a lower apoapsis or increasing control authority by increasing $L / D$.

Another aerocapture trade reflected in these results is that, in general, more is gained from the lift-up trajectories than from liftdown. From inspection of the example in Fig. 3 , it is clear that one appealing configuration is a lift-modulated orbiter with a ballistic probe trajectory. The ballistic probe trajectory could be truly passive, such as for a simple penetrator probe mission, or it could apply lift- or drag-modulation to the ballistic trajectory for the purpose of accommodating uncertainties. Passive impactor or penetrator probes are 
already excellent candidates for co-delivery due to their simplicity and small size, and so this configuration stands out as a promising mission architecture for multiple reasons.

\section{Representative Scenario}

The previous section explores a large trade space for probe and orbiter co-delivery by considering numerous point designs. Each of these trajectories is only passively controlled (full-lift-up or -down, or ballistic) and has no accounting for random uncertainties. To further demonstrate the fundamental feasibility of the proposed co-delivery method from a flight mechanics standpoint, this section more closely examines a single representative mission scenario. A nominal scenario is defined that makes use of bank-angle modulation lift control for both the probe and the orbiter. Representative uncertainties are then applied and their effect quantified through a Monte Carlo analysis.

\section{A. Methodology}

A general co-delivery scenario involves two vehicles, an aerocapture orbiter and a direct-entry probe, each of which may implement some closed-loop guidance to control their atmospheric flight. As noted in Sec. II.C, a particular scenario of interest would involve a passive ballistic probe; because this vehicle would be significantly simpler than an entry vehicle using active guidance and control, it may be a better fit for ride-along probe missions. Thus, three vehicles are considered in this section: a guided orbiter that performs aerocapture, a guided probe following a direct-entry trajectory, and a passive ballistic probe that follows a similar direct-entry trajectory.

Mars is chosen as the destination for this representative example scenario. The trajectories are simulated using the same equations of motion as in Sec. II.A, with the addition of the $J_{2}$ zonal term in the spherical harmonics gravity model, where $J_{2}=0.001964$ [51]. For the orbiter and guided probe, bank-angle modulation is used as the method of control, which updates the orientation of the lift vector about the velocity vector without changing the angle of attack. This method is selected here for its flight heritage on the MSL and Mars 2020 missions [36,52], but note that other control approaches, such as drag-skirt jettison or direct-force control, would also be applicable.

Mode 1 of the Fully Numerical Predictor-Corrector Aerocapture Guidance (FNPAG) scheme developed by Lu et al. [11] is implemented for the orbiter. This guidance algorithm assumes a bang-bang structure to lift modulation, wherein the vehicle uses Brent's method [53] to numerically predict a switching time from a lift-up angle $0 \leq \sigma_{i}<90$ deg to a lift-down angle $90 \mathrm{deg}<\sigma_{d} \leq 180 \mathrm{deg}\left(\sigma_{i}\right.$ is called $\sigma_{0}$ in [11], renamed here to distinguish from the Fully Numerical Predictor-Corrector Entry Guidance (FNPEG) variable of the same name). This bang-bang structure targets the desired apoapsis while minimizing the total $\Delta V$ required for the periapsis raise and apoapsis correction maneuvers. The following simplifying assumptions are made for the purpose of this demonstration:

1) Only longitudinal guidance is implemented, meaning a final apoapsis radius is targeted while ignoring the final inclination or wedge angle. Lateral guidance is normally achieved separately from the modulation of the bank-angle magnitude through periodic bank reversals [11]. Assuming no plane change is desired during aerocapture, the feasibility of achieving the desired apoapsis under uncertainties can be approximately assessed without considering the lateral guidance component, even though for any real aerocapture mission the lateral logic is an important part of the guidance scheme.

2) The initial bank angle is assumed to be $\sigma_{i}=0 \mathrm{deg}$, and the initial guess for the final bank angle (which is updated during phase 2 of FNPAG) is assumed to be $\sigma_{d}=150 \mathrm{deg}$. Note that with $\sigma_{i}=0 \mathrm{deg}$, there is neither lateral force nor lateral control authority during phase 1 , but bank reversals could be performed during phase 2 .

3) The guidance is run at a rate of $1 \mathrm{~Hz}$ and the bank-angle is updated instantaneously, neglecting the effect of a finite roll rate and acceleration for the vehicle.
4) No additional trajectory constraints are imposed, such as limits on peak heat flux or g-load, because the value of those limits would be strongly mission dependent.

5) No atmospheric estimation model is included in the guidance implementation, as this was found to be unnecessary to demonstrate fundamental feasibility for this scenario.

These simplifying assumptions are appropriate here because this section presents a proof-of-concept demonstration; a dedicated mission analysis would iteratively tune $\sigma_{d}$, potentially assume a larger value for $\sigma_{i}$, implement bank reversals and a finite roll rate and acceleration, and so on.

A similar approach is taken for the guided probe, which implements FNPEG, developed by Lu [54]. FNPEG assumes the bankangle magnitude profile is a linear function of $e$, as shown in Eq. (3), where $e$ is the negative of the specific orbital energy, as given in Eq. (4). The value of $\sigma_{0}$ is then updated with each guidance call to target a desired range and energy, where the desired energy is computed by applying Eq. (4) to the desired radial distance and inertial velocity at the final time. Note that, because the target values for radius and velocity are combined into a single constraint, FNPEG can result in small altitude and velocity errors, but in many applications (such as targeting range at parachute deploy), this is acceptable [54]. At each guidance call, FNPEG uses the golden-section method to minimize the error function [Eq. (5)] [53], where $s\left(e_{f}\right)$ is the predicted great-circle range at the target energy, and $s_{f}^{*}$ is the target final range. The value of $s\left(e_{f}\right)$ is predicted numerically by including range $s$ (in radians) in the equations of motion as $\dot{s}=V \cos (\gamma) / r$ and propagating until the target energy is reached [54], where $V$ and $\gamma$ are both planet-relative values. Similar assumptions are made here as for FNPAG. Lateral guidance is again neglected for the same reasons, instantaneous bank-angle updates are assumed with guidance run at a rate of $1 \mathrm{~Hz}$, and no additional trajectory constraints are imposed. A value of $\sigma_{f}=60 \mathrm{deg}$ is used for this study. As with FNPAG, these assumptions are made for the sake of a proof-of-concept demonstration, and a more detailed mission analysis would tune $\sigma_{f}$, implement finite roll rate and acceleration, etc.

$$
\begin{gathered}
|\sigma(e)|=\sigma_{0}+\frac{e-e_{0}}{e_{f}-e_{0}}\left(\sigma_{f}-\sigma_{0}\right) \\
e=\frac{\mu}{r}-\frac{v^{2}}{2} \\
f\left(\sigma_{0}\right)=\frac{1}{2}\left[s\left(e_{f}\right)-s_{f}^{*}\right]^{2}
\end{gathered}
$$

\section{B. Nominal Scenario}

A nominal scenario is constructed starting from the results shown in Fig. 4. As seen from the full-lift-up plot in Fig. 4, a lifting vehicle with $\beta=130 \mathrm{~kg} \cdot \mathrm{m}^{-2}$ can achieve aerocapture with a low apoapsis from an EFPA of $-12 \mathrm{deg}$. As seen in the ballistic plot, a probe with $\beta=35 \mathrm{~kg} \cdot \mathrm{m}^{-2}$ can follow a direct-entry trajectory from the same EFPA, either as a ballistic probe or a lifting vehicle dedicating some or all of its control authority to mitigating uncertainties. The orbiter ballistic coefficient was chosen to be similar to that of MSL [36], and the probe ballistic coefficient to be similar to that of the Mars Microprobe capsules [47]. As in the trade study, the guided vehicles have a lift-to-drag ratio of $L / D=0.25$, whereas the passive probe is ballistic $(L / D=0)$. As before, the entry state is defined at the atmospheric interface altitude with a planet-relative entry velocity of $V_{R, 0}=6 \mathrm{~km} \cdot \mathrm{s}^{-1}$, with a due-east initial heading angle at $18.38^{\circ}$ latitude. The nominal values of key parameters are listed in Table $\underline{2}$ in the "Mean" column.

For the orbiter, the target final orbit is defined to be a $250 \mathrm{~km}$ altitude circular orbit. By running an FNPAG trajectory once with no dispersions (a perfect predictor), the switching time required to reach this apoapsis from the entry state described previously is found to be approximately $152.6 \mathrm{~s}$. This nominal trajectory results in a nominal total $\Delta V$ cost of $74 \mathrm{~m} \cdot \mathrm{s}^{-1}$, as shown in Table $\underline{3}$. This total $\Delta V$ is 
computed as the sum of the $\Delta V$ for a periapsis raise maneuver performed at the initial apoapsis $\left(\Delta V_{1}\right)$ and the $\Delta V$ for a subsequent apoapsis correction maneuver performed at the new periapsis $\left(\Delta V_{2}\right)$. This cost is computed as in Eq. (6), where $r_{a}$ and $r_{p}$ are the apoapsis and periapsis radii of the post-atmospheric state, respectively, and $r_{a}^{*}$ and $r_{p}^{*}$ are the desired apoapsis and periapsis radii, respectively:

$$
\begin{aligned}
\Delta V=\Delta V_{1}+\Delta V_{2} & =\sqrt{2 \mu}\left|\left[\sqrt{\frac{1}{r_{a}}-\frac{1}{r_{a}+r_{p}^{*}}}-\sqrt{\frac{1}{r_{a}}-\frac{1}{r_{a}+r_{p}}}\right]\right| \\
+\sqrt{2 \mu} & {\left[\sqrt{\frac{1}{r_{p}^{*}}-\frac{1}{r_{a}^{*}+r_{p}^{*}}}-\sqrt{\frac{1}{r_{p}^{*}}-\frac{1}{r_{a}+r_{p}^{*}}}\right] \mid }
\end{aligned}
$$

For the guided probe, the target altitude and velocity are set to $15 \mathrm{~km}$ and $300 \mathrm{~m} \cdot \mathrm{s}^{-1}$, respectively, and the target range is approximately $700.8 \mathrm{~km}$. This target state corresponds to a Mach number of 1.3 and a dynamic pressure of $175 \mathrm{~Pa}$, where the speed of sound on Mars is found by interpolating from the table provided in [35]. Depending on the specific mission design, the final state targeted by FNPEG could represent parachute deployment, retrorocket ignition, or simply a shift to some other guidance method as the entry capsule continues down to the surface. The main purpose here is to give FNPEG something to aim for so that the effect of uncertainties can be understood, rather than to design a full EDL sequence. With these target values and a perfect predictor, FNPEG computes an initial bank-angle magnitude of approximately $\sigma_{0}=139.3 \mathrm{deg}$. This nominal trajectory results in zero range error, but has altitude and velocity errors of $441 \mathrm{~m}$ and $-5.5 \mathrm{~m} \cdot \mathrm{s}^{-1}$, respectively. As mentioned in Sec. III.A, here FNPEG undershoots the target velocity and overshoots the target altitude in such a way that the final energy is still correct, but these errors are relatively small and could also be further reduced by optimization of the reference trajectory. The discrepancy is mainly notable because a bias can be expected in the results under uncertainty due to these nonzero errors for the nominal trajectory.

Lastly, the passive ballistic probe has no target state because it has no variable control authority during atmospheric flight. To compare results with the guided probe, the trajectory of the passive probe is always terminated at $15 \mathrm{~km}$ altitude, and the errors are defined as differences compared to the nominal velocity and range values at this altitude: $353.1 \mathrm{~m} \cdot \mathrm{s}^{-1}$ and $735.0 \mathrm{~km}$, respectively. Note that this means there are no performance results for altitude for the passive probe. This corresponds to a Mach number of 1.6 and a dynamic pressure of $242 \mathrm{~Pa}$.

The nominal trajectories for these three vehicles are shown in Fig. $\underline{8}$, where the blue dot shows the point along the trajectory of the orbiter where it switches from lift-up $\left(\sigma_{i}\right)$ to lift-down $\left(\sigma_{d}\right)$. The

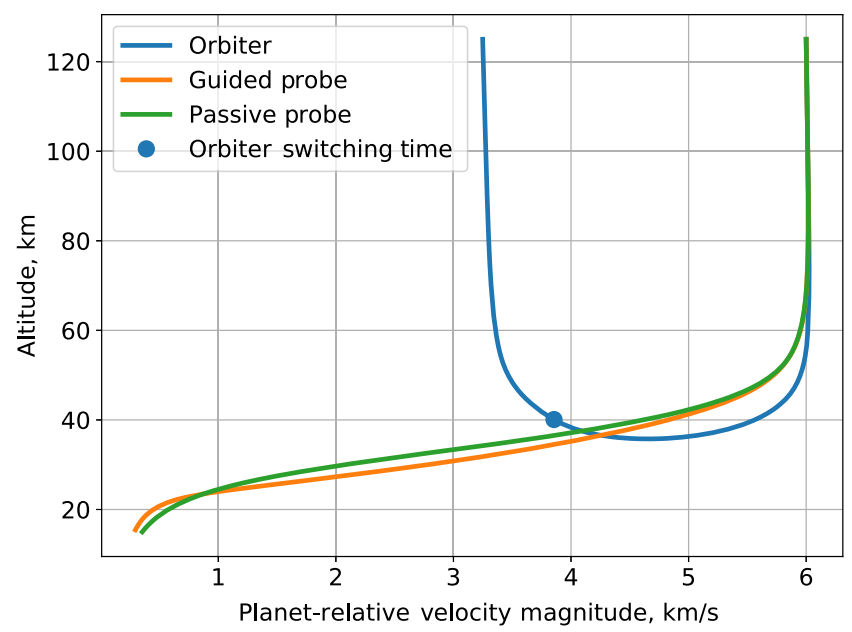

Fig. 8 Nominal trajectories for the orbiter, guided lifting probe, and passive ballistic probe.
Table 2 Input dispersions

\begin{tabular}{lcc}
\hline \hline Parameter & Mean & Dispersions \\
\hline EFPA & $-12 \mathrm{deg}$ & $3 \sigma=0.2 \mathrm{deg}$ \\
Entry velocity & $6 \mathrm{~km} \cdot \mathrm{s}^{-1}$ & $3 \sigma=10 \mathrm{~m} \cdot \mathrm{s}^{-1}$ \\
Orbiter $\beta$ & $130 \mathrm{~kg} \cdot \mathrm{m}^{-2}$ & $\pm 5 \%$ \\
Probe $\beta$ & $35 \mathrm{~kg} \cdot \mathrm{m}^{-2}$ & $\pm 5 \%$ \\
Orbiter $L / D$ & 0.25 & $\pm 5 \%$ \\
Guided probe $L / D$ & 0.25 & $\pm 5 \%$ \\
Density & Mars-GRAM 2010 & Mars-GRAM 2010 \\
\hline \hline
\end{tabular}

orbiter trajectory and either of the two probe trajectories constitute one representative scenario on Mars; a similar process could be followed for any of the feasible regions of the trade space identified in Sec. II.

\section{Performance Under Uncertainty}

Having designed nominal trajectories for a guided orbiter, guided probe, and passive probe, the performance of these vehicles is assessed under representative uncertainties in the entry state, vehicle aerodynamics, and atmospheric density. A 1500-trial Monte Carlo analysis is performed. The mean and dispersions for each randomized input are listed in Table 2 , where $3 \sigma=X$ indicates a Gaussian distribution with zero mean and standard deviation $\sigma$, and $\pm Y \%$ indicates the bounds for a uniform distribution relative to the mean. The input parameter is computed by adding the mean and a dispersion value randomly generated from the associated probability distribution.

Variation in the entry state is simulated by independently normally dispersing the planet-relative EFPA and planet-relative entry velocity magnitude. Because the orbiter and probe are assumed to share a delivery state, the same randomly selected entry state is used for all three vehicles for each trial. The numerical predictor component of FNPAG and FNPEG is given perfect state knowledge, including of the entry state, and so the EFPA and velocity dispersions represent guidance performance under a range of initial conditions, as opposed to performance with an imperfect predictor or error between the navigated and true states. The EFPA dispersion is set equal to the delivery requirement for MSL, and the entry velocity dispersion is set to five times the MSL requirement for the navigation knowledge accuracy used for EDL guidance system initialization [23]. The larger entry velocity dispersion is used in this study to generate a wider range of potential entry states for illustrative purposes.

Uncertainty in the aerodynamic properties of the vehicles is modeled by independently uniformly dispersing ballistic coefficient and $L / D$. Because the orbiter and probe are separate vehicles, their aerodynamic properties are dispersed separately. However, because the passive probe is included for direct comparison to the guided probe, its ballistic coefficient is always set equal to the actual value of the ballistic coefficient of the guided probe; there is no dispersion on the $L / D$ of the passive probe because it always equals zero. Unlike the entry state, the numerical predictor guidance always uses the nominal values for $\beta$ and $L / D$, whereas the true state is propagated using the dispersed values for each trial, resulting in an imperfect predictor. The uniform $\pm 5 \%$ dispersion for these vehicle parameters represents modeling uncertainty associated with computational fluid dynamics analysis and ballistic range testing, and is based on values used in previous studies [55].

Finally, atmospheric density variability on Mars is modeled using Mars-GRAM 2010, which has a built-in capability to output randomly perturbed correlated density profiles in a Monte Carlo sense [41]. Because the orbiter and probe would arrive simultaneously and experience the same atmosphere, the same dispersed density profile is used for all three vehicles in each trial. Differences in the density encountered at a given altitude due to different paths through the atmosphere are assumed negligible for this study. As with the aerodynamics dispersions, the guidance algorithm always uses the nominal density profile in its predictions, whereas the true state is propagated according to the dispersed density profile. The Mars-GRAM 2010 settings are 
Table 3 Performance results under uncertainty

\begin{tabular}{lccc}
\hline \hline Parameter & Nominal & Mean & Standard deviation \\
\hline Orbiter apoapsis error, $\mathrm{km}$ & 0 & 30.87 & 65.05 \\
Orbiter total $\Delta V$ cost, $\mathrm{m} \cdot \mathrm{s}^{-1}$ & 73.73 & 86.75 & 16.85 \\
Guided probe altitude error, $\mathrm{m}$ & 441.1 & 466.9 & 338.4 \\
Guided probe range error, $\mathrm{km}$ & 0 & -0.1821 & 1.076 \\
Guided probe velocity error, $\mathrm{m} \cdot \mathrm{s}^{-1}$ & -5.486 & -5.840 & 4.250 \\
Passive probe range error, $\mathrm{km}$ & 0 & 0.1483 & 9.984 \\
Passive probe velocity error, $\mathrm{m} \cdot \mathrm{s}^{-1}$ & 0 & 0.1006 & 11.45 \\
\hline \hline
\end{tabular}

generally kept at their default values, including a perturbation scale of 1 and solar radio flux at $10.7 \mathrm{~cm}$ of $68 \mathrm{sfu}$ [41], using the date of 18 February 2021.

The results of this 1500-trial Monte Carlo analysis are summarized in Table 3, and histograms of error and cost parameters are shown in Figs. $9-12$. The purpose of this analysis is to demonstrate feasibility for this mission scenario and to compare the performance of the guided and passive probes, not to precisely estimate the performance metrics of the vehicles. By numerically examining the convergence as the number of trials was increased, the quantities of interest listed in Table 3 are found to converge to within roughly $\pm 5 \%$. The mean range error for both probes and mean velocity error for the passive probe are exceptions to this statement because, as quantities with nominal values of zero and mean values near zero, their percent errors are poorly behaved. The guided probe range error converges to within roughly $0.05 \mathrm{~km}$, the passive probe range error to roughly $0.2 \mathrm{~km}$, and the passive probe velocity error to roughly $0.5 \mathrm{~m} \cdot \mathrm{s}^{-1}$.

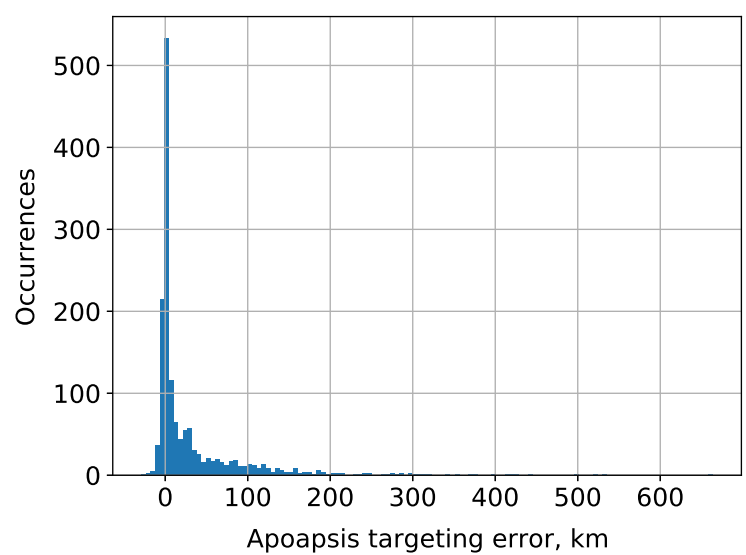

a) Histogram of apoapsis error for orbiter
The orbiter achieves aerocapture with a $100 \%$ success rate despite a relatively large range in entry states, although some cases do significantly overshoot the desired apoapsis, as seen in Fig. 9. Note that Fig. 9 b is a close-up view of Fig. 9a to better see those high-error cases. These errors also lead to a positive skew in the total $\Delta V$ results shown in Fig. 10a, with the worst cases exceeding twice the nominal cost. Note that the total $\Delta V$ results are centered nearly one standard deviation above the nonzero nominal value. Although these errors are significant, they are not unexpected, considering the relatively large entry state dispersions and the use of an imperfect predictor in the guidance algorithm. The large overshoot cases are often a result of saturation in phase 2 of FNPAG, meaning the vehicle flies full lift down but is still unable to sufficiently reduce its energy, resulting in an apoapsis that is higher than desired. With better tuning of the $\sigma_{d}$ parameter, robustness could be improved and a reduction in the high-error cases may be achieved. The performance could also be improved by adding some adaptive atmospheric estimation capability to the guidance implementation $[11,56]$.

The altitude performance for the guided probe is shown in Fig. 10b, and the range and velocity performance is compared between the guided and passive probes in Figs. 11 and 12, respectively. As expected, the altitude and velocity errors for the guided probe are centered near the nonzero nominal error values. Although the specific requirements for this delivery accuracy would be mission dependent, in general FNPEG shows good performance. Particularly notable for this study is the comparison of range and velocity errors between the guided and passive probes. As expected, the passive probe does perform much worse than the guided probe; in terms of standard deviation, the passive probe has roughly double the velocity error and

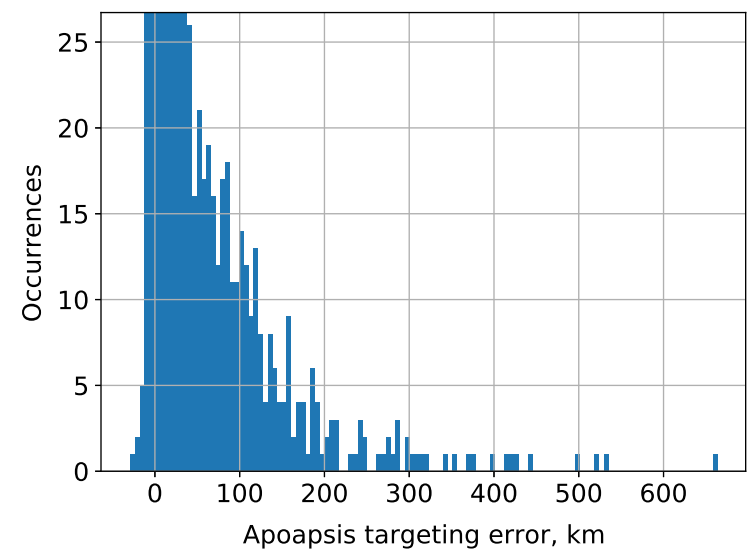

b) Zoomed-in view of Fig. 9a

Fig. 9 Apoapsis results for orbiter.

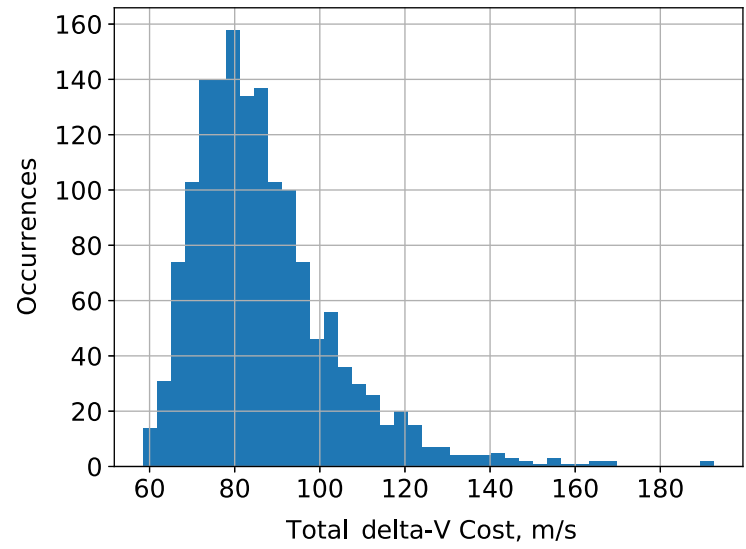

a) Histogram of total $\Delta V$ cost for orbiter

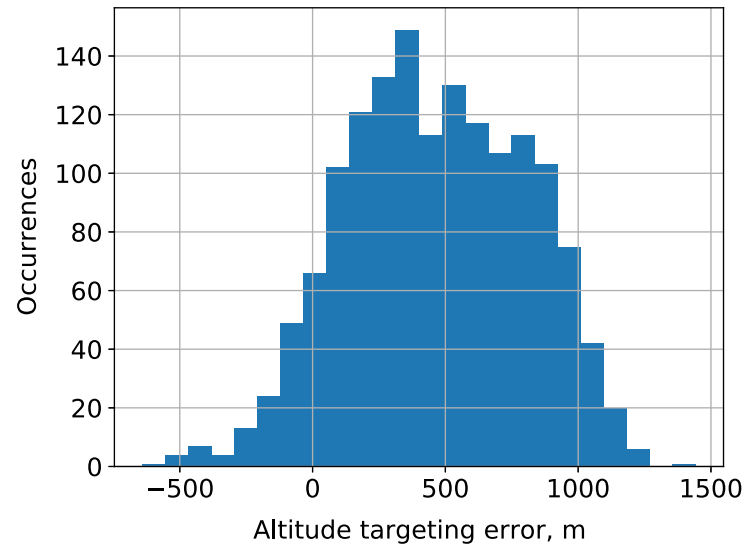

b) Histogram of altitude error for guided probe

Fig. $10 \Delta V$ cost for orbiter and altitude error for guided probe. 


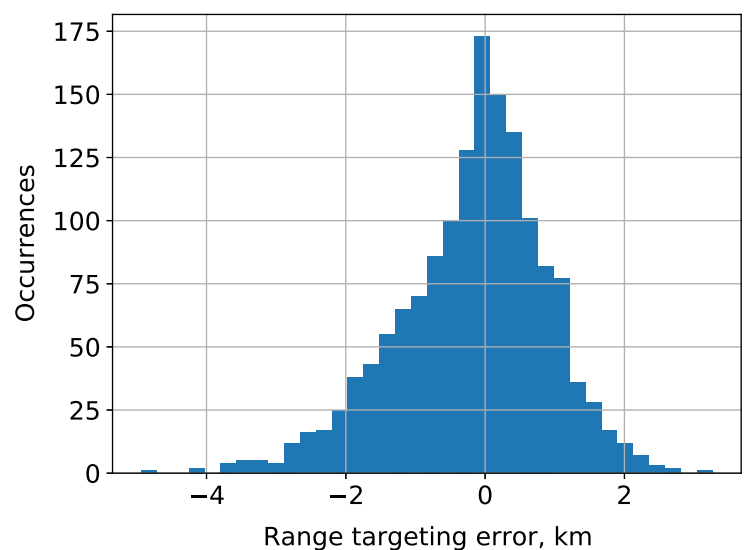

a) Guided probe

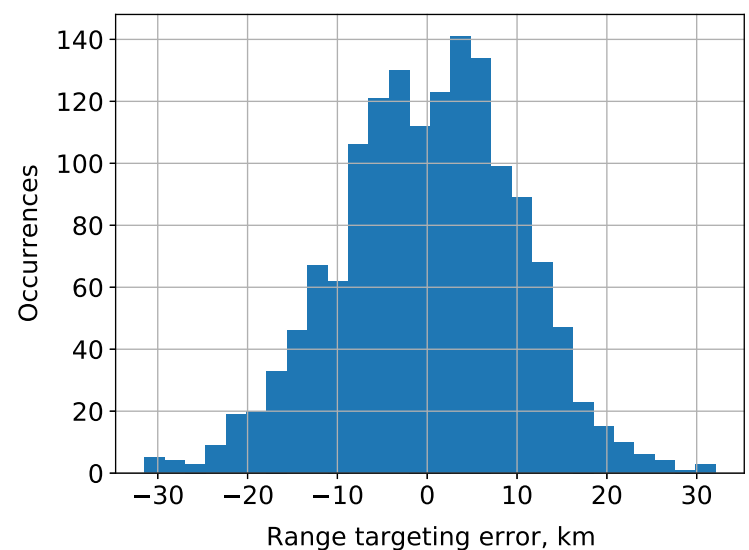

b) Passive probe

Fig. 11 Target range error histograms for guided and passive probes.

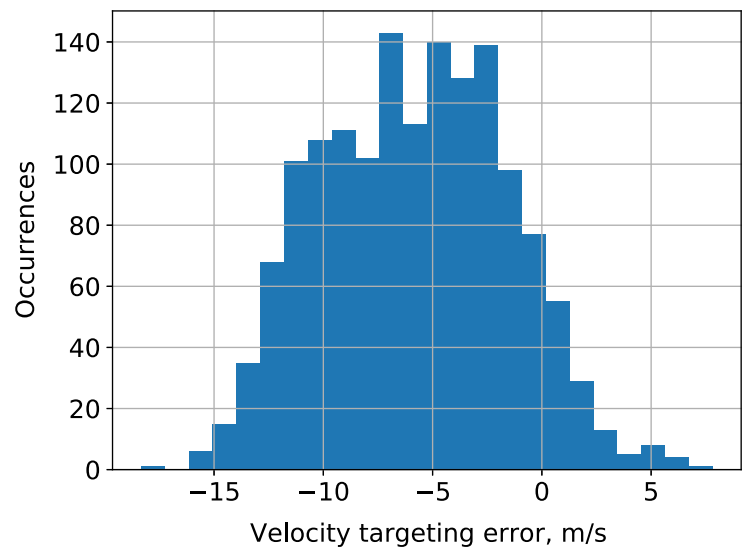

a) Guided probe

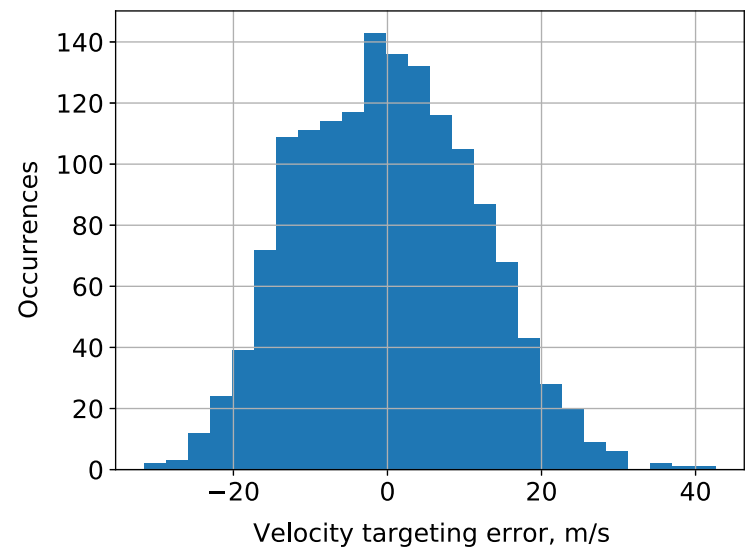

b) Passive probe

Fig. 12 Target velocity error histograms for guided and passive probes.

roughly an order of magnitude more range error compared to the guided probe. That said, a delivery uncertainty on the order of $\pm 50 \mathrm{~km}$ range and $\pm 25 \mathrm{~m} \cdot \mathrm{s}^{-1}$ velocity at $15 \mathrm{~km}$ altitude may well be acceptable for some applications. For example, if the probe were a small secondary ride-along payload targeting a broad surface region either by parachute or as an impactor, perhaps these error ranges would be sufficient.

The Monte Carlo analysis results are included to demonstrate two main conclusions. First, aerocapture and direct-entry trajectories from the same entry state are feasible even under significant navigation, vehicle, and atmospheric dispersions if the orbiter is provided some control authority and closed-loop guidance capability. Second, the probe can target a specific final state if also provided control authority and closed-loop guidance, although the final state dispersions for a passive ballistic probe may already be sufficient for some applications. The performance results presented in this section are intended to serve as a proof of concept for one representative scenario at Mars under uncertainty.

\section{Conclusions}

The proposed co-delivery method is an architecture for SmallSat ride-along missions to interplanetary destinations. This co-delivery architecture is shown to be feasible for wide ranges of vehicle and trajectory parameters at Earth, Mars, Venus, Titan, and Neptune, subject to mission-specific heating and g-load constraints that are quantified across this trade space. An example scenario is developed using FNPAG and FNPEG closed-loop guidance for the orbiter and probe, respectively, and the performance of the vehicles under uncertainty is shown to be adequate through a Monte Carlo analysis. Based on the trade space analysis and the uncertainty quantification results, a passive ballistic impactor or penetrator probe as a secondary mission on an orbiter delivered by a lift-modulated aerocapture trajectory is shown to be a particularly promising configuration. A number of challenges remain for implementation, including separation design, timing and observation geometry, packaging, and tight volume and mass constraints.

\section{Acknowledgments}

This work was supported by a NASA Space Technology Research Fellowship. The first author thanks Jay McMahon and Soumyo Dutta for help with the Fully Numerical Predictor-Corrector Aerocapture Guidance and the Fully Numerical Predictor-Corrector Entry Guidance materials.

\section{References}

[1] Halt, T., Wieger, A., Boensch, N., Dolgopolov, A., Smith, P., and Hernandez, A., "Smallsats by the Numbers 2019," Bryce Space and Technology, Alexandria, VA, 2019.

[2] Poghosyan, A., and Golkar, A., "CubeSat Evolution: Analyzing CubeSat Capabilities for Conducting Science Missions," Progress in Aerospace Sciences, Vol. 88, Jan. 2017, pp. 59-83. https://doi.org/10.1016/j.paerosci.2016.11.002 
[3] Norton, C. D., Pellegrino, S., and Johnson, M., "Small Satellites: A Revolution in Space Science," Keck Inst. for Space Studies, California Inst. of Technology, Pasadena, CA, July 2014.

[4] Mercer, C. R., "Small Satellite Missions for Planetary Science," Proceedings of the Small Satellite Conference, Upcoming Missions, SSC19-WKV-06, 2019, pp. 1-6, https://digitalcommons.usu.edu/ smallsat/2019/all2019/102/.

[5] Asmar, S. W., and Matousek, S., Mars Cube One (MarCO): Shifting the Paradigm in Relay Deep Space Operation, AIAA, Reston, VA, 2016, pp. 1-7.

[6] Cassell, A. M., Smith, B. P., Wercinski, P. F., Ghassemieh, S. M., Hibbard, K. E., Nelessen, A. P., and Cutts, J. A., "ADEPT, A Mechanically Deployable Re-Entry Vehicle System, Enabling Interplanetary CubeSat and Small Satellite Missions," Proceedings of the Small Satellite Conference, Advanced Technologies I, SSC18-XII-08, 2018, pp. 1-8, https://digitalcommons.usu.edu/smallsat/2018/all2018/265/.

[7] Barba, N., Komarek, T., Woolley, R., Giersch, L., Stamenkovié, V., Gallagher, M., and Edwards, C. D., "Mars Small Spacecraft Studies: Overview," 2019 IEEE Aerospace Conference, IEEE, New York, 2019, pp. $1-10$. https://doi.org/10.1109/AERO.2019.8741735

[8] Gage, P., Mahzari, M., Peterson, K., Ellerby, D., and Venkatapathy, E., "Technology Readiness Assessment for HEEET TPS," 16th International Planetary Probe Workshop, 2019, pp. 1-19.

[9] Putnam, Z. R., and Braun, R. D., "Drag-Modulation Flight-Control System Options for Planetary Aerocapture," Journal of Spacecraft and Rockets, Vol. 51, No. 1, 2014, pp. 139-150. https://doi.org/10.2514/1.A32589

[10] Deshmukh, R. G., Spencer, D. A., and Dutta, S., "Investigation of Direct Force Control for Aerocapture at Neptune," Acta Astronautica, Vol. 175, Oct. 2020 , pp. $375-386$ https://doi.org/10.1016/j.actaastro.2020.05.047

[11] Lu, P., Cerimele, C. J., Tigges, M. A., and Matz, D. A., "Optimal Aerocapture Guidance," Journal of Guidance, Control, and Dynamics, Vol. 38, No. 4, 2015, pp. 553-565. https://doi.org/10.2514/1.G000713

[12] Heidrich, C. R., and Braun, R. D., "Aerocapture Trajectory Design in Uncertain Entry Environments," AIAA SciTech 2020 Forum, AIAA Paper 2020-1741, Jan. 2020, pp. 1-21. https://doi.org/10.2514/6.2020-1741

[13] Halder, A., and Bhattacharya, R., "Dispersion Analysis in Hypersonic Flight During Planetary Entry Using Stochastic Liouville Equation," Journal of Guidance, Control, and Dynamics, Vol. 34, No. 2, 2011, pp. 459-474. https://doi.org/10.2514/1.51196

[14] Ridderhof, J., and Tsiotras, P., "Stochastic Atmosphere Modeling for Risk Adverse Aerocapture Guidance," 2020 IEEE Aerospace Conference, IEEE, New York, 2020, pp. 1-7. https://doi.org/10.1109/AERO47225.2020.9172724

[15] Albert, S. W., Doostan, A., and Schaub, H., "Finite-Dimensional Density Representation for Aerocapture Uncertainty Quantification," AIAA SciTech 2021 Forum, AIAA Paper 2021-0932, Jan. 2021, pp. 1-17. https://doi.org/10.2514/6.2021-0932

[16] Smith, B. P., Tanner, C. L., Mahzari, M., Clark, I. G., Braun, R. D., and Cheatwood, F. M., "A Historical Review of Inflatable Aerodynamic Decelerator Technology Development," 2010 IEEE Aerospace Conference, IEEE, New York, 2010, pp. 1-18. https://doi.org/10.1109/AERO.2010.5447013

[17] Hughes, S., Cheatwood, F., Dillman, R., Wright, H., DelCorso, J., and Calomino, A., "Hypersonic Inflatable Aerodynamic Decelerator (HIAD) Technology Development Overview," 21 st AIAA Aerodynamic Decelerator Systems Technology Conference and Seminar, AIAA Paper 2011-2524, May 2011, pp. 1-24. https://doi.org/10.2514/6.2011-2524

[18] Spilker, T. R., Adler, M., Arora, N., Beauchamp, P. M., Cutts, J. A., Munk, M. M., Powell, R. W., Braun, R. D., and Wercinski, P. F., "Qualitative Assessment of Aerocapture and Applications to Future Missions," Journal of Spacecraft and Rockets, Vol. 56, No. 2, 2019, pp. 536-545. https://doi.org/10.2514/1.A34056

[19] Austin, A., "Enabling and Enhancing Science Exploration Across the Solar System: Aerocapture Technology for SmallSat to Flagship Missions," Bulletin of the American Astronomical Society, Vol. 54, No. 4, March 2021, https://baas.aas.org/pub/2021n4i057.

[20] Dutta, S., "Aerocapture as an Enhancing Option for Ice Giants Missions," Bulletin of the American Astronomical Society, Vol. 53, No. 4, March 2021, https://baas.aas.org/pub/2021n4i046.

[21] Hofstadter, M., Simon, A., Reh, K., Elliott, J., Niebur, C., and Colangeli, L., "Ice Giants Pre-Decadal Study Final Report," NASA TR JPL D100520, June 2017.
[22] Hall, J. L., Noca, A. N., and Bailey, R. W., "Cost-Benefit Analysis of the Aerocapture Mission Set," Journal of Spacecraft and Rockets, Vol. 42, No. 2, 2005, pp. 309-320. https://doi.org/10.2514/1.4118

[23] Martin-Mur, T. J., Kruizinga, G. L., Burkhart, P. D., Abilleira, F., Wong, M. C., and Kangas, J. A., "Mars Science Laboratory Interplanetary Navigation," Journal of Spacecraft and Rockets, Vol. 51, No. 4, 2014, pp. 1014-1028. https://doi.org/10.2514/1.A32631

[24] Colin, L., "The Pioneer Venus Program," Journal of Geophysical Research: Space Physics, Vol. 85, No. A13, 1980, pp. 7575-7598. https://doi.org/10.1029/JA085iA13p07575

[25] O'Neil, W. J., The Three Galileos: The Man, the Spacecraft, the Telescope, Vol. 220, Springer, Dordrecht, The Netherlands, 1997, pp. 75-94.

[26] Kazeminejad, B., Atkinson, D. H., Pérez-Ayũcar, M., Lebreton, J.-P., and Sollazzo, C., "Huygens' Entry and Descent Through Titan's Atmosphere-Methodology and Results of the Trajectory Reconstruction," Planetary and Space Science, Vol. 55, No. 13, 2007, pp. 1845-1876. https://doi.org/10.1016/j.pss.2007.04.013

[27] Klesh, A. T., Baker, J., and Krajewski, J., "MarCO: Flight Review and Lessons Learned," Proceedings of the Small Satellite Conference, SSC19-III-04, 2019, pp. 1-6, https://digitalcommons.usu.edu/ smallsat/2019/all2019/102/.

[28] Albert, S. W., Braun, R. D., and Schaub, H., "AeroDrop: Prospects and Challenges for Co-Delivery of Probe and Orbiter via Aerocapture," AAS/AIAA Astrodynamics Specialist Conference, AAS Paper 20-718, Univelt, Inc., San Diego, CA, 2020, pp. 2275-2293.

[29] Vinh, N. X., Busemann, A., and Culp, R. D., Hypersonic and Planetary Entry Flight Mechanics, Univ. of Michigan Press, Ann Arbor, MI, 1980, pp. 26-27, Chap. 2.

[30] Dormand, J. R., and Prince, P. J., "A Family of Embedded Runge-Kutta Formulae," Journal of Computational and Applied Mathematics, Vol. 6 , No. 1, 1980, pp. 19-26. https://doi.org/10.1016/0771-050X(80)90013-3.

[31] Graves, C. A., and Harpold, J. C., "Apollo Experience Report—Mission Planning for Apollo Entry," NASA TN D-6725, 1972.

[32] Lockwood, M. K., Starr, B. R., Paulson, J. W., Jr., Kontinos, D. A., Chen, Y., Laub, B., Olejniczak, J., Wright, M. J., Takashima, N., Justus, C. G., and Duval, A. L., "Systems Analysis for a Venus Aerocapture Mission,” NASA TM-2006-214291, 2006.

[33] Lockwood, M. K., Edquist, K. T., Starr, B. R., Hollis, B. R., Hrinda, G. A., Bailey, R. W., Hall, J. L., Spilker, T. R., Noca, M. A., O'Kongo, N., and Haw, R. J., "Aerocapture Systems Analysis for a Neptune Mission," NASA TM-2006-214300, 2006.

[34] McNamara, L. W., and Rea, J. R., "Definition of the Design Entry Trajectory and Entry Flight Corridor for the NASA Orion Exploration Mission 1 Using an Integrated Approach and Optimization," 2014 American Astronautical Society Guidance and Control Conference, AAS Paper 14-094, Univelt, Inc., San Diego, CA, 2014, pp. 529-542.

[35] Justus, C. G., and Braun, R. D., "Atmospheric Environments for Entry, Descent and Landing (EDL)," 5th International Planetary Probes Workshop and Short Course, 2007, pp. 1-37. https://ntrs.nasa.gov/ citations/20070032693 [retrieved 7 July 2021].

[36] Way, D., Powell, R., Chen, A., Steltzner, A., San Martin, M., Burkhart, P., and Mendeck, G., "Mars Science Laboratory: Entry, Descent, and Landing System Performance," IEEE Aerospace Conference Proceedings, IEEE, New York, 2006, pp. 1-19.

[37] Trainer, M. G., Wong, M. H., McConnochie, T. H., Franz, H. B., Atreya, S. K., Conrad, P. G., Lefévre, F., Mahaffy, P. R., Malespin, C. A., Manning, H. L., Martín-Torres, J., Martínez, G. M., McKay, C. P., Navarro-Gonzílez, R., Vicente-Retortillo, A., Webster, C. R., and Zorzano, M.-P., "Seasonal Variations in Atmospheric Composition as Measured in Gale Crater, Mars," Journal of Geophysical Research: Planets, Vol. 124, No. 11, 2019, pp. 3000-3024.

https://doi.org/10.1029/2019JE006175

[38] Girija, A. P., Saikia, S. J., Longuski, J. M., Bhaskaran, S., Smith, M. S., and Cutts, J. A., "Feasibility and Performance Analysis of Neptune Aerocapture Using Heritage Blunt-Body Aeroshells," Journal of Spacecraft and Rockets, Vol. 57, No. 6, 2020, pp. 1186-1203. https://doi.org/10.2514/1.A34719

[39] Lindal, G. F., Lyons, J. R., Sweetnam, D. N., Eshleman, V. R., Hinson, D. P., and Tyler, G. L., "The Atmosphere of Neptune: Results of Radio Occultation Measurements with the Voyager 2 Spacecraft," Geophysical Research Letters, Vol. 17, No. 10, 1990, pp. 1733-1736. https://doi.org/10.1029/GL017i010p01733

[40] Leslie, F., and Justus, C., "The NASA Marshall Space Flight Center Earth Global Reference Atmospheric Model-2010 Version,” NASA TM-2011-216467, 2011. 
[41] Justh, H. L., "Mars Global Reference Atmospheric Model 2010 Version: Users Guide," NASA TM-2014-217499, 2014.

[42] Justh, H. L., "Venus Global Reference Atmospheric Model," NASA TR M17-6248, 2017, https://ntrs.nasa.gov/citations/20170012290 [retrieved 7 July 2021].

[43] Justh, H. L., and Hoffman, J., "Titan Global Reference Atmospheric Model (Titan-GRAM): User Guide," NASA TM-20205006805, 2020.

[44] Justh, H. L., and Hoffman, J., "Neptune Global Reference Atmospheric Model (Neptune-GRAM): User Guide," NASA TM-20205001193, 2020.

[45] Sutton, K., and Graves, R. A., "A General Stagnation-Point ConvectiveHeating Equation for Arbitrary Gas Mixtures," NASA TR R-376, 1971.

[46] Heidrich, C., Roelke, E. E., Albert, S. W., and Braun, R. D., "Comparative Study of Lift- and Drag-Modulation Control Strategies for Aerocapture," 43rd Annual AAS Guidance, Navigation and Control Conference, AAS Paper 20-074, Univelt, Inc., San Diego, CA, 2020, pp. 627-642.

[47] Braun, R. D., Mitcheltree, R. A., and Cheatwood, F. M., "Mars Microprobe Entry-to-Impact Analysis," Journal of Spacecraft and Rockets, Vol. 36, No. 3, 1999, pp. 412-420. https://doi.org/10.2514/2.3461.

[48] Goodwin, J., and Wegner, P., "Evolved Expendable Launch Vehicle Secondary Payload Adapter-Helping Technology Get to Space," AIAA Space 2001 Conference and Exposition, AIAA Paper 2001-4701, Aug. 2001, pp. 1-7.

https://doi.org/10.2514/6.2001-4701

[49] Barba, N. J., Komarek, T. A., Stamekovic, V., Giersh, L. R., Woolley, R. C., and Edwards, C. D., "Small Spacecraft Capabilities for Mars Exploration," Ninth International Conference on Mars, Lunar and Planetary Inst., LPI Contrib. No. 2089, 2019, https://www.hou.usra.edu/meetings/ ninthmars2019/pdf/6341.pdf.
[50] Albert, S. W., and Braun, R. D., "Conceptual Development of AeroDrop: Aerocapture and Direct Entry for Two Spacecraft on a Common Approach Trajectory," AIAA SciTech 2020 Forum, AIAA Paper 20201737, Jan. 2020, pp. 1-20. https://doi.org/10.2514/6.2020-1737

[51] Vallado, D. A., Fundamentals of Astrodynamics and Applications, 4th ed., Microcosm Press, Hawthorne, CA, 2013, pp. 1041-1042.

[52] Brugarolas, P., "Guidance, Navigation and Control for the Entry, Descent, and Landing of the Mars 2020 Mission," 40th Annual AAS Guidance and Control Conference, AAS Paper 17-031, Univelt, Inc., San Diego, CA, 2017, pp. 81-92, https://trs.jpl.nasa.gov/handle/2014/ 46389.

[53] Brent, R. P., Algorithms for Minimization Without Derivatives, Courier Corp., Englewood Cliffs, NJ, 2013.

[54] Lu, P., "Entry Guidance: A Unified Method," Journal of Guidance, Control, and Dynamics, Vol. 37, No. 3, 2014, pp. 713-728. https://doi.org/10.2514/1.62605

[55] Austin, A., Nelessen, A., Strauss, B., Ravich, J., Jesick, M., Venkatapathy, E., Beck, R., Wercinski, P., Aftosmis, M., Wilder, M., Allen, G., Braun, R., Werner, M., and Roelke, E., "SmallSat Aerocapture to Enable a New Paradigm of Planetary Missions," 2019 IEEE Aerospace Conference, IEEE, New York, 2019, pp. 1-20. https://doi.org/10.1109/AERO.2019.8742220

[56] Roelke, E., Hattis, P., and Braun, R., "Improved Atmospheric Estimation for Aerocapture Guidance," AAS/AIAA Astrodynamics Specialist Conference, AAS Paper 19-725, Univelt, Inc., San Diego, CA, 2019, pp. 2415-2430.

K. T. Edquist Associate Editor 